\title{
Energy-preserving integrators and the structure of B-series
}

by

\author{
Elena Celledoni ${ }^{1}$, Robert I McLachlan ${ }^{2}$, Brynjulf Owren, ${ }^{*}$ and GRW \\ Quispel $^{3}$
}

\author{
PREPRINT \\ NUMERICS NO. 5/2009
}

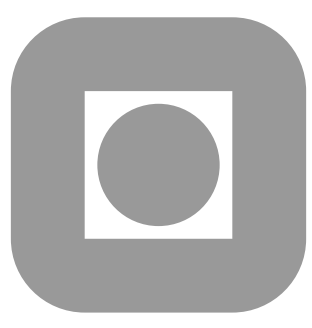

\author{
NORWEGIAN UNIVERSITY OF \\ SCIENCE AND TECHNOLOGY \\ TRONDHEIM, NORWAY
}

This report has URL http://www. math.ntnu.no/preprint/numerics/2009/N5-2009.pdf

Address: Department of Mathematical Sciences, Norwegian University of Science and

Technology, N-7491 Trondheim, Norway.

\footnotetext{
${ }^{1}$ Department of Mathematical Sciences, NTNU, 7491 Trondheim, Norway

${ }^{2}$ Institute of Fundamental Sciences, Massey University, Palmerston North, New Zealand

${ }^{3}$ Mathematics Department, La Trobe University, VIC 3086, Australia
} 



\title{
Energy-preserving integrators and the structure of B-series
}

\author{
Elena Celledoni, Robert I McLachlan, Brynjulf Owren, ${ }^{*}$ and GRW Quispel"
}

June 16, 2009

\begin{abstract}
B-series are a powerful tool in the analysis of Runge-Kutta numerical integrators and some of their generalizations ("B-series methods"). A general goal is to understand what structure-preservation can be achieved with B-series and to design practical numerical methods that preserve such structures. B-series of Hamiltonian vector fields have a rich algebraic structure that arises naturally in the study of symplectic or energy-preserving B-series methods and is developed in detail here. We study the linear subspaces of energy-preserving and Hamiltonian modified vector fields which admit a B-series, their finite-dimensional truncations, and their annihilators. We characterize the manifolds of B-series conjugate to Hamiltonian and conjugate to energy-preserving and describe the relationships of all these spaces.
\end{abstract}

\section{Introduction: the Average Vector Field integrator}

The study of the behaviour of first integrals under numerical integration has a long history. In the most general setting their error grows linearly with time, but for special methods or special differential equations the error can grow sublinearly, can be bounded, or can be zero (apart from round-off error; see [12] for a discussion of these cases for energy behaviour.) Amongst methods that preserve first integrals, we can distinguish between cases in which the integral lies in a class whose structure ensures that the integral is preserved automatically and cases in which the preservation is enforced. Examples of the former include automatic preservation of linear integrals by all Runge-Kutta methods [21], and automatic preservation of quadratic integrals by some (the symplectic) Runge-Kutta methods [7]. The user does not even need to know of the existence of the integral for it to be preserved! Lie group integrators, which preserve a homogeneous space as a submanifold, can also be put in this class, although the user does need to know the group actions that generate motion on the submanifold. An example of the latter is the projection method: first generate any approximation to the desired point, and then project it on to the desired level set of the integral. While this approach is still

\footnotetext{
${ }^{\S}$ Department of Mathematical Sciences, NTNU, 7491 Trondheim, Norway

"Institute of Fundamental Sciences, Massey University, Palmerston North, New Zealand

"Mathematics Department, La Trobe University, VIC 3086, Australia
} 
widely used when preservation of the integral is absolutely critical, it has some drawbacks: the projection can destroy other properties of the integrator, and the domain in which the projected point exists can shrink to zero as critical points of the integral are approached. Where possible, 'automatic' preservation is preferred.

A very large class of one-step methods are those that have B-series, i.e., Taylor series in the time step in which each term is a sum of elementary differentials of the vector field. Runge-Kutta, multiderivative Runge-Kutta, some exponential integrators, some linearizationpreserving integrators [15], and some generating function methods have B-series. B-series methods preserve linear invariants and affine symmetries automatically. If it can be shown that no B-series apart from the exact solution have a certain property, then a very large class of methods is ruled out and one will have to look elsewhere. Volume-preservation is one example $[1,6]$, and preservation of arbitrary first integrals of arbitrary vector fields is another [5]. If, on the other hand, there are B-series with a desired property, this does not ensure the existence of B-series methods, for a numerical method has to be finitely described and computable.

With this background it came as a great surprise that there are B-series methods that conserve energy in Hamiltonian systems. The first to be discovered was the Average Vector Field (AVF) method first written down in [14] and identified as energy-preserving and as a B-series method in [19]: for the differential equation

$$
\dot{x}=f(x), \quad x \in \mathbb{R}^{n},
$$

the AVF method is the map $x \mapsto x^{\prime}$ defined by

$$
\frac{x^{\prime}-x}{h}=\int_{0}^{1} f\left(\xi x^{\prime}+(1-\xi) x\right) \mathrm{d} \xi .
$$

Note that the conserved quantity is arbitrary, and does not appear explicitly in the definition of the method (or in its B-series), cf. Theorem 1 below. This is possible because of the special relationship between the energy integral and the vector field that exists just for Hamiltonian systems. Energy preservation is 'automatic': the user does not even need to know that the specified vector field $f$ is Hamiltonian, but if it is, its energy will be preserved.

Theorem 1. The AVF method is a B-series method, is affine-covariant [16], self-adjoint, and of order 2. When $f$ is Hamiltonian with respect to a constant symplectic structure, i.e., when $f=\Omega^{-1} \nabla H$ with $\Omega$ a nonsingular, antisymmetric matrix, the AVF method preserves the Hamiltonian $H: \mathbb{R}^{n} \rightarrow \mathbb{R}$. It is pseudo-symplectic of order 2 (but not 3) and conjugatepseudo-symplectic of order 4 (but not 5).

We refer to [4] for the proof, although the demonstration of energy-preservation bears repeating as it illustrates how constancy of $\Omega$ plays a crucial role. Let $y=\xi x^{\prime}+(1-\xi) x$ :

$$
\begin{aligned}
H\left(x^{\prime}\right)-H(x) & =\int_{0}^{1} \nabla H(y)^{\top}\left(x^{\prime}-x\right) \mathrm{d} \xi \\
& =h\left(\int_{0}^{1} \nabla H(y) \mathrm{d} \xi\right)^{\top}\left(\int_{0}^{1} f(y) \mathrm{d} \xi\right) \\
& =h\left(\int_{0}^{1} \nabla H(y) \mathrm{d} \xi\right)^{\top} \Omega^{-1}\left(\int_{0}^{1} \nabla H(y) \mathrm{d} \xi\right) \\
& =0 .
\end{aligned}
$$


Also note that the exact solution can be written in a form similar to (2), but with the integral being along the orbit connecting $x$ and $x^{\prime}$ instead of along a straight line.

In contrast to projection methods, the equations defining the (implicit) AVF method always have a solution. We show this by casting it as a continuous-stage Runge-Kutta method with $a(\xi, \eta)=\xi$ and $b(\xi)=1$.

Definition 1. Let $a$ and $b$ be such that the linear maps $L^{\infty}([0,1]) \rightarrow L^{\infty}([0,1]), k \mapsto$ $\int_{0}^{1} a(\xi, \eta) k(\eta) \mathrm{d} \eta$ and $L^{\infty}([0,1]) \rightarrow \mathbb{R}, k \mapsto \int_{0}^{1} b(\eta) k(\eta)$ are bounded. The method $x \mapsto x^{\prime}$ defined by

$$
\begin{aligned}
k(\xi) & =f\left(x+h \int_{0}^{1} a(\xi, \eta) k(\eta) \mathrm{d} \eta\right), \quad \xi \in[0,1] \\
x^{\prime} & =x+h \int_{0}^{1} b(\xi) k(\xi) \mathrm{d} \xi
\end{aligned}
$$

is called a continuous-stage Runge-Kutta method.

The following theorem is an instance of a more general theorem of Butcher [2] covering convergence of a very wide class of Runge-Kutta-like methods, and which ensures the existence of unique solutions to the equations of the AVF method for sufficiently small step sizes.

Theorem 2. Let $f: \mathbb{R}^{n} \rightarrow \mathbb{R}^{n}$ be continuous and satisfy a Lipschitz condition with constant L. If

$$
h<\frac{1}{L \sup _{\xi \in[0,1]} \int_{0}^{1}|a(\xi, \eta)| \mathrm{d} \eta}
$$

then there exists a unique solution of (4).

For ordinary differential equations

$$
\dot{y}=f(y),
$$

where $y$ lies in a vector space, we consider numerical integrators $y \mapsto \Phi_{h}(y)$ that have B-series

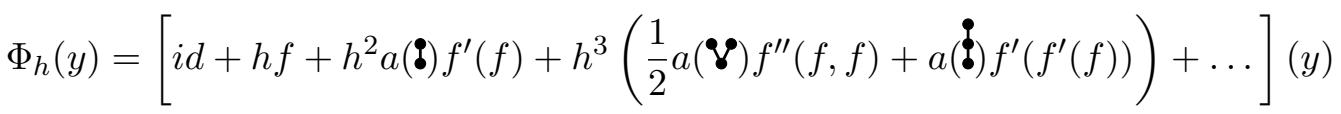

$$
\begin{aligned}
& =\sum_{\tau \in T \cup \emptyset} h^{|\tau|} \frac{a(\tau)}{\sigma(\tau)} F(\tau)(y)
\end{aligned}
$$

where $T$ is the set of rooted trees. We write $T^{n}$ for the set of rooted trees with $n$ vertices, and $|t|=n$ if $t \in T^{n}$ (thus $T=\cup_{n=1}^{\infty} T^{n}$ ) and let

$$
\begin{aligned}
\mathcal{T}^{n} & =\operatorname{span}\left(T^{n}\right) \\
\mathcal{T} & =\operatorname{span}(T)=\bigoplus_{n=1}^{\infty} \mathcal{T}^{n} .
\end{aligned}
$$

The integrator has a modified vector field $\tilde{f}(y)$ that also has a B-series, but one with leading coefficient 0 :

$$
\Phi_{h}(y)=\exp (h \tilde{f}(y)), \quad \tilde{f}=\sum_{\tau \in T} h^{|\tau|-1} \frac{b(\tau)}{\sigma(\tau)} F(\tau)(y) .
$$


We principally work with B-series of modified vector fields; in contrast to B-series of maps, they are linear objects which can largely be understood order-by-order.

The rest of the paper is organized as follows: In Section 2 we present the linear subspaces of $B$-series of Hamiltonian and energy-preserving vector fields. We characterize the graded components of these subspaces in terms of dimensions, bases and annihilators. In Section 3 we prove several new results for B-series of vector fields which are conjugate to Hamiltonian or energy-preserving vector fields. We shall in particular discuss how all the subspaces are related to each other and we shall present results related to the dimensions of the various graded components and their intersections.

\section{Energy-preserving and Hamiltonian B-series}

Let $\Omega$ be an antisymmetric, invertible $n \times n$ matrix. It defines a symplectic inner product on $\mathbb{R}^{n}$ by $(u, v):=u^{\top} \Omega v$. The Hamiltonian vector field with Hamiltonian function $H$ is defined by $X_{H}:=\Omega^{-1} \nabla H$. These have two key properties: their flow is energy-preserving (because $\left.\dot{H}=X_{H}(H)=(\nabla H)^{\top} \Omega^{-1} \nabla H=0\right)$ and symplectic, i.e., tangent vectors to solutions, that obey the linearized differential equation $\dot{u}=X_{H}^{\prime}(x(t)) u$, preserve the symplectic inner product, $\frac{d}{d t}(u(t), v(t))=0$. (The entire theory presented in this paper also applies to the case of Poisson systems with constant Poisson structure, i.e., to the systems $\dot{x}=J \nabla H$ with $J$ a constant, not necessarily invertible matrix.)

When $f$ is a Hamiltonian vector field, its elementary differentials have certain special properties: certain linear combinations of them are Hamiltonian, or have first integral $H$, for example. These define linear subspaces of the vector space over rooted trees. These subspaces inherit the linear- and Lie-algebraic structure induced by that of the elementary differentials that remains even when the original vector field $f$ is 'forgotten' and one works only with trees. Here we explore that structure.

We extend the elementary differential $F$ to $\mathcal{T}$ by linearity, e.g. $F(a \bullet+b \boldsymbol{\bullet})=a F(\bullet)+b F(\boldsymbol{\bullet})$.

Definition 2. The energy-preserving subspace (of order $n$ ) is defined by

$$
\mathcal{T}_{H}^{n}:=\left\{t \in \mathcal{T}^{n}: F(t) \text { has first integral } H \text { when } f=\Omega^{-1} \nabla H\right\}
$$

The Hamiltonian subspace (of order $n$ ) is defined by

$$
\mathcal{T}_{\Omega}^{n}:=\left\{t \in \mathcal{T}^{n}: F(t) \text { is Hamiltonian w.r.t. } \Omega \text { when } f=\Omega^{-1} \nabla H\right\}
$$

We have

$$
\begin{aligned}
& \mathcal{T}_{H}=\bigoplus_{n=1}^{\infty} \mathcal{T}_{H}^{n} \\
& \mathcal{T}_{\Omega}=\bigoplus_{n=1}^{\infty} \mathcal{T}_{\Omega}^{n}
\end{aligned}
$$

and we let $\mathcal{F}_{(H, \Omega)}^{n}:=F\left(\mathcal{T}_{(H, \Omega)}^{n}\right)$. That is, an element of $\mathcal{F}$ is a B-series of a modified vector field.

We briefly recall some well known definitions to be used in the sequel. A forest is an unordered, possible empty, collection of trees, where each tree can appear an arbitrary number of times. Let $\bar{T}$ be the set of forests. A recursive representation of trees is given as follows: 
$t \in T$ is either the one node tree $t=\bullet=[\emptyset]$ or $t=\left[t_{1}, \ldots, t_{n}\right]$ obtained by joining the roots of each tree $t_{i}$ to a new common root. Thus $|t|=1+\sum_{i}\left|t_{i}\right|$. We shall also make use of the map $B_{-}: T \rightarrow \bar{T}$ defined as $B_{-}(\bullet)=\emptyset$ and $B_{-}\left(\left[t_{1}, \ldots, t_{n}\right]\right)=t_{1} \ldots t_{n}$. The Butcher product between two trees $u=\left[u_{1}, \ldots, u_{n}\right]$ and $v$ is defined as $u \circ v=\left[u_{1}, \ldots, u_{n}, v\right]$. Note that $\circ$ is non-commutative. In fact, this product induces an equivalence relation on $T$, the smallest equivalence relation satisfying $u \circ v \sim v \circ u$ for every $u, v \in T[10,11]$. Two equivalent trees have the same graph, and differ only in the position of the root; thus they also have the same order. For two equivalent trees, $\theta$ and $\tau, \kappa(\theta, \tau)$ is the number of times the root must be shifted in order to obtain $\theta$ from $\tau$. Each equivalence class is called a free tree, and we denote by $F T^{n}$ the set of all free trees of order $n$. Let $\pi(\tau)$ be the free tree (equivalence class) to which $\tau \in T$ belongs. A free tree is called superfluous if it contains an element of the form $u \circ u$ for some $u \in T$. All other free trees are called nonsuperfluous.

$\mathcal{T}_{H}^{n}$ and $\mathcal{T}_{\Omega}^{n}$ are linear subspaces of $\mathcal{T}^{n}$. We seek to understand these subspaces, their annihilators, their dimensions, and their relationships. Let $|V|$ be the dimension of the vector space $V$. One subspace, $\mathcal{T}_{\Omega}^{n}$, is already well understood; see for example Hairer et al. [11] and Calvo and Sanz-Serna [3] for the analogue for maps.

Theorem 3. A basis of $\mathcal{F}_{\Omega}^{n}$ is given by the Hamiltonian vector fields of the elementary Hamiltonians associated with the nonsuperfluous free trees of order $n$. A basis of the annihilator $\operatorname{Ann}\left(\mathcal{T}_{\Omega}^{n}\right)$ of $\mathcal{T}_{\Omega}^{n}$ is given by ${ }^{1}$

$$
\left\{(u \circ v)^{*}+(v \circ u)^{*}: u, v \in T,|u|+|v|=n\right\},
$$

so that Hamiltonian B-series of the form $\sum_{|t|=n} \frac{b(t)}{\sigma(t)} t \in \mathcal{T}^{n}$, satisfy

$$
b(u \circ v)+b(v \circ u)=0, \quad u, v \in T,|u|+|v|=n .
$$

The dimension of $\mathcal{T}_{\Omega}^{n}$ is given by

$$
\left|\mathcal{T}_{\Omega}^{n}\right|= \begin{cases}\left|F T^{n}\right|-\left|T^{n / 2}\right|, & n \text { even } \\ \left|F T^{n}\right|, & n \text { odd }\end{cases}
$$

Example 1. There are 4 trees of order 4 , for which the standard basis is: $\frac{1}{6} \boldsymbol{V}, \dot{\boldsymbol{V}}, \frac{1}{2}$, !. There is 1 nonsuperfluous free tree of order 4 , namely $\pi(\boldsymbol{Y})$. The combination of trees associated with its Hamiltonian vector field is $3 \boldsymbol{Y}-\boldsymbol{V},\left(6\left(\frac{1}{2}\right)-6\left(\frac{1}{6} \boldsymbol{Y}\right)\right.$ in the standard basis), which is a basis for $\mathcal{T}_{\Omega}^{4}$. A basis for the annihilator of this subspace is obtained by

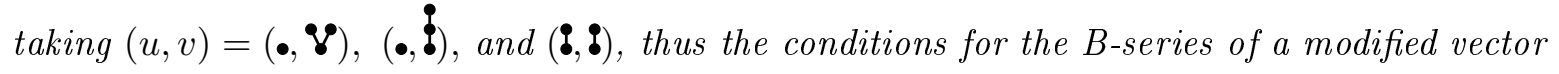
field to be Hamiltonian at order 4 are

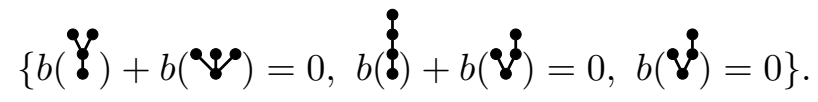

The conditions to be energy-preserving - i.e., the annihilator of $\mathcal{T}_{H}^{n}$-were first given in [10], and a spanning set of $\mathcal{T}_{H}^{n}$ is given in [19]. The analogue for maps is studied in [5].

A set of energy-preserving trees was given in [19].

\footnotetext{
${ }^{1}$ here we denote by $t^{*}$ the element of the dual space such that $\left\langle t^{*}, \frac{t^{\prime}}{\sigma\left(t^{\prime}\right)}\right\rangle=\delta_{t, t^{\prime}}$ for any $t \in T$.
} 
Theorem 4. Let

$$
S:=\left\{\left[t_{1},\left[t_{2}, \ldots,\left[t_{m}, \bullet\right] \cdots\right]+(-1)^{m}\left[t_{m},\left[\ldots,\left[t_{2},\left[t_{1}, \bullet\right] \cdots\right]: t_{j} \in \bar{T}\right\},\right.\right.\right.
$$

then $S \subseteq \mathcal{T}_{H}$.

Proof. Any element of $S$ is of the form $t+(-1)^{m} \hat{t}$ with

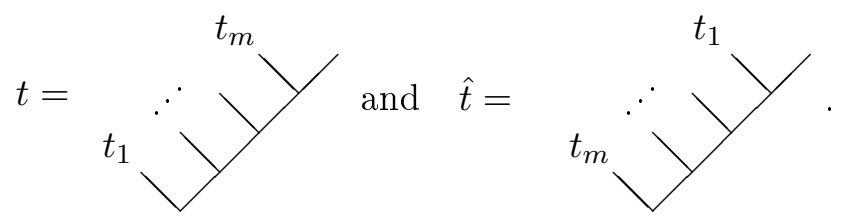

It follows from the chain rule that $H$ is preserved by the flow of a vector field $g$ if and only if $H^{\prime}(g(y))=0$ along integral curves $y$. In particular, we must prove that this is true for the vector field $F(t)+(-1)^{m} F(\hat{t})$. From the definition of the elementary Hamiltonian associated to a tree [11, sec. IX.9.2], we see that $H^{\prime}(F(t))=H([t])$. Using the root-shifting property $H(u \circ v)=-H(v \circ u)$ for all trees $u$ and $v$, the root of $[t]$ can be moved to an adjacent vertex incurring a change of sign. We shift the root up $m+1$ places until it reaches the designated - in $[t]=\left[\left[t_{1},\left[t_{2}, \ldots,\left[t_{m}, \bullet\right] \cdots\right]\right]\right.$. The resulting tree is $[\hat{t}]$, thus $\kappa(t, \hat{t})=m+1$, and we find that $H([\hat{t}])=(-1)^{m+1} H([t])$. So, again using the definition of the elementary Hamiltonian we conclude that $H^{\prime}\left(F(t)+(-1)^{m} F(\hat{t})\right)=0$, thus proving that the vector field $F(t)+(-1)^{m} F(\hat{t})$ preserves $H$.

Example 2. The B-series of the modified vector field of the AVF method (2) can be written as follows, where we have replaced each elementary differential by its tree and grouped terms into energy-preserving pairs:

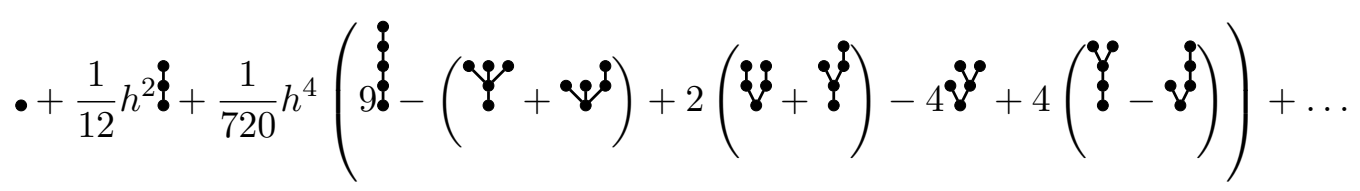

This can be calculated by using the explicit formula the B-series of the map (2) found in [4] and recursively calculating the B-series of the modified vector field as in [11, ch. IX.9.1].

Theorem 5. (i) A basis for the annihilator $\operatorname{Ann}\left(\mathcal{T}_{H}^{n}\right)$ of $\mathcal{T}_{H}^{n}$ can be indexed over the nonsuperfluous elements of $F T^{n+1}$ as follows:

$$
\left\{\sum_{\substack{\tau \in \pi^{-1}(\phi) \\ \tau=[\bar{\tau}]}}(-1)^{\kappa\left(\tau_{0}, \tau\right)} \frac{1}{\sigma(\tau)} \bar{\tau}^{*}: \phi \in F T^{n+1}, \phi \text { nonsuperfluous }\right\}
$$

where $\tau_{0}$ is a designated element of $\pi^{-1}(\phi) .{ }^{2}$ The sum is taken over all trees $\tau \in \pi^{-1}(\phi)$ having precisely one subtree.

\footnotetext{
${ }^{2}$ In Murua [17] a canonical representative for $\pi^{-1}(\phi)$ is constructed. Note also that apart from a change of sign, the annihilator element is independent of the choice of $\tau_{0}$.
} 
(ii) The dimension of $\mathcal{T}_{H}^{n}$ is given by

$$
\left|\mathcal{T}_{H}^{n}\right|=\left|\mathcal{T}^{n}\right|-\left|\mathcal{T}_{\Omega}^{n+1}\right|
$$

(iii) The set $S$ contains a basis of $\mathcal{T}_{H}^{n}$.

Proof. (i) and (ii) are special cases of [11, Ch IX], Lemma 9.11 and Corollary 9.12. (iii) For a nonsuperfluous free tree $\phi \in F T^{n+1}$ the condition to be satisfied by

$$
\sum_{\bar{\tau} \in T^{n}} \frac{b(\bar{\tau})}{\sigma(\bar{\tau})} \bar{\tau} \quad \text { is } \quad \sum_{\substack{\tau \in \pi^{-1}(\phi) \\ \tau=[\bar{\tau}]}}(-1)^{\kappa\left(\tau_{0}, \tau\right)} \frac{b(\bar{\tau})}{\sigma(\tau)}=0 .
$$

Note that the same tree cannot appear in two different energy preserving conditions, because for any tree $\tau \in T^{n}$, there is at most one nonsuperfluous free tree $\phi \in F T^{n+1}$ such that $[\tau] \in \pi^{-1}(\phi) . \mathcal{T}^{n}$ is thus a direct sum of subspaces, each corresponding to at most one free tree in $F T^{n+1}$. Therefore we can construct a basis for $\mathcal{T}_{H}^{n}$ by taking a basis for the solutions of each energy-preserving condition independently. If the nonsuperfluous free tree has $m$ leaves up to symmetries, the condition involves $m$ trees. If $m=1$, there are no energy-preserving elements in the subspace spanned by that tree. If $m>1$, a basis for this condition can be obtained by choosing any $m-1$ pairs of trees, spanning independent subspaces, and solving the condition (10) in the 2-dimensional space of that pair. This yields basis elements expressed as linear combinations of distinct pairs of trees. To see that these take the form of (7), grasp the two leaves whose deletion yielded the selected trees and pull them apart. The taut part is the backbone of (7). Placing one leaf on the ground and cutting it off defines the first term in (7), while turning the whole tree upside down to put the other leaf on the ground and cutting if off yields the second term in (7) To check the coefficients, recall that the conditions (8) are defined using (6) that involves symmetry factors in the coefficients.

The basis built thus far involves all trees except those $t$ for which $[t]$ is superfluous. All such $t$ are energy-preserving and can be included in the basis and, following the construction of the previous paragraph, take the form (7) with $n$ even and $t_{i}=t_{n+1-i}$ so that the two trees in (7) coincide.

Note that the first term in (7) is just a representation of an arbitrary tree, with the spine being the path from the root to any leaf. The second tree is identical to the first iff $[t]$ is superfluous. Put another way, each superfluous free tree generates a singleton energypreserving tree.

Conversely, the second tree will cancel the first iff the free tree $[t]$ is symmetric, that is, a root can be chosen so that all the vertices at the same distance from the root have the same number of edges; equivalently, taking any leaf of $[t]$ to be the root yields the same rooted tree. Symmetric trees are given by $\left(\ldots\left(\bullet^{n_{1}}\right)^{n_{2}} \ldots\right)^{n_{k}}$ where $t^{n}:=[t, \ldots, t](n t$ 's). The bushy tree is $\bullet^{n}$, the tall tree is $t_{\text {tall }}=\left(\ldots\left(\bullet^{1}\right)^{1} \ldots\right)^{1}$, and the $n$-legged starfish is $t_{\text {tall }}^{n}$. Trees with $[t]$ symmetric can never appear in an energy-preserving B-series unless they are superfluous, for this is precisely the annihilation condition (8) associated with $[t]$. The only superfluous symmetric trees are the tall trees with an even number of vertices. For the bushy trees, this is another way of showing that energy-preserving B-series integrators must satisfy all the quadrature order conditions [4] and hence that no Runge-Kutta method is energy-preserving for arbitrary Hamiltonians. 
It is a curious fact that the elements of any basis of the Hamiltonian B-series contain many terms, while their annihilator has a basis in which each element consists of 2 terms, while for the energy-preserving B-series the situation is reversed: they have a basis in which each element contains $\leq 2$ terms, while the elements of their annihilator contain many terms.

Because the only Hamiltonian B-series that preserves the energy is the exact flow of the differential equation ([5],[11], IX.9.15), the energy-preserving and the Hamiltonian trees scarcely intersect:

$$
\mathcal{T}_{\Omega} \cap \mathcal{T}_{H}=\bullet
$$

\section{Conjugate-to-energy preserving and conjugate-to-Hamiltonian B-series}

We now consider B-series that are conjugate (by a B-series) to an energy-preserving or a Hamiltonian B-series. Such B-series do not form linear spaces, but some of their propertiese.g., their dimension - can be described using two new linear spaces that we call $\mathcal{T}_{\widetilde{H}}^{n}$ and $\mathcal{T}_{\widetilde{\Omega}}^{n}$. See the Appendix for a geometric description of these spaces.

Recall that the integrator $\Phi$ is said to be conjugate-symplectic if there exists a map $\Psi$ such that $\Psi \Phi \Psi^{-1}$ is symplectic. Equivalently, $\Phi$ preserves not the original symplectic form $\Omega$ but a modified symplectic form $\left(\Psi^{-1}\right)^{*} \Omega$. If $\Phi$ has a B-series then it is natural to require that $\Psi$ also has a B-series. The conditions on $\Phi$ (or its modified vector field) that ensure conjugate-symplecticity have been derived up to order 5 in $[11,20]$.

We would like to describe the modified vector fields that are conjugate by B-series to Hamiltonian ones, i.e., to eliminate the conjugacy from the description. It turns out to be most convenient to do this by considering the conjugacy to be fixed up to some order and variable thereafter. We will see that this is reasonable because, given a modified vector field that is conjugate to Hamiltonian, the conjugacy is determined, essentially uniquely, order by order.

We now need to use Lie brackets of vector fields. We write these as $\llbracket f, g \rrbracket$ to distinguish them from the root-grafting operation $[t]$. The Lie bracket of vector fields induces a Lie bracket on $\mathcal{T}: \llbracket t_{1}, t_{2} \rrbracket$ is given the sum of the grafts of $t_{1}$ onto each vertex of $t_{2}$, minus the sum of the grafts of $t_{2}$ onto each vertex of $t_{1}$. Because the Hamiltonian and energy-preserving vector fields form Lie algebras, $\mathcal{F}_{H}$ and $\mathcal{F}_{\Omega}$ (resp. $\mathcal{T}_{H}$ and $\mathcal{T}_{\Omega}$ ) form graded Lie subalgebras of $\mathcal{F}$ (resp. $\mathcal{T}$ ). For the following lemmas we provide proofs using vector fields and purely combinatorial proofs in terms of trees.

Let $c$ be the B-series whose flow conjugates the flow of $\tilde{f}$, i.e.,

$$
\mathrm{e}^{c} \mathrm{e}^{\tilde{f}} \mathrm{e}^{-c}=\mathrm{e}^{\hat{f}}
$$

where $\hat{f}$ is Hamiltonian. We call $c$ the conjugacy. Rearranging,

$$
\mathrm{e}^{\tilde{f}}=\mathrm{e}^{-c} \mathrm{e}^{\hat{f}} \mathrm{e}^{c} .
$$

Thus, the conjugate-Hamiltonian B-series are given by

$$
\left\{\log \left(\mathrm{e}^{-c} e^{\hat{f}} \mathrm{e}^{c}\right): c \in \mathcal{T}, \hat{f} \in \mathcal{T}_{\Omega}\right\} .
$$

This is equal to

$$
\left\{\hat{f}-\llbracket c, \hat{f} \rrbracket+\frac{1}{2} \llbracket c, \llbracket c, \hat{f} \rrbracket \rrbracket-\ldots: c \in \mathcal{T}, \hat{f} \in \mathcal{T}_{\Omega}\right\}
$$


We now make three restrictions: first, we consider only B-series of integrators, i.e. $\hat{f}(\bullet)=$ $\tilde{f}(\bullet)=1$. Second, we consider only non-Hamiltonian conjugacies $c$. The definition of nonHamiltonian is arbitrary, but turns out to be immaterial.

Definition 3. Let $\mathcal{T}_{\Omega}^{\prime}$ be any complement of $\mathcal{T}_{\Omega}$ in $\mathcal{T}$. Let $\mathcal{T}_{H}^{\prime}$ be any complement of $\mathcal{T}_{H}$ in $\mathcal{T}$.

For example, we could choose $\mathcal{T}_{H}^{3 \prime}=\operatorname{span}(\boldsymbol{V})$, or any other 1-dimensional subspace of $\mathcal{T}^{3}$ independent of $\mathcal{T}_{H}^{3}=\operatorname{span}(\$)$. We take $c \in \mathcal{T}_{\Omega}^{\prime}$.

The motivation for this restriction is that the conjugate of a Hamiltonian B-series by a Hamiltonian B-series is another Hamiltonian B-series, so we expect that including Hamiltonian terms in the conjugacy will not be very useful (but see Theorem 10 below).

Third, instead of allowing the conjugacy $c$ to range over all non-Hamiltonian B-series in (11), we will take its terms of order $<n-1$ to be fixed; and instead of allowing $\hat{f}$ to range over all Hamiltonian B-series, we will take its terms of order $<n$ to be fixed. This is a useful restriction because it will turn out that, given $\tilde{f}$, the $c$ that conjugates it to Hamiltonian will be determined uniquely order-by-order.

Lemma 6. $\mathrm{ad}$. is $1-1$ on $\mathcal{T}^{n}$ for $n>1$.

Proof. Suppose not. Then there are distinct trees $t_{1}, t_{2}$ such that $\llbracket t_{1}, \bullet \rrbracket=\llbracket t_{2}, \bullet \rrbracket$. Then $\llbracket F\left(t_{1}-t_{2}\right), f \rrbracket=0$, i.e., $f$ has the nontrivial symmetry $F\left(t_{1}-t_{2}\right)$ for all $f$. But there are $f$ 's with no nontrivial symmetries, a contradiction.

Proof. (A tree-based version). We write $\tau \in \mathcal{T}^{n}$ in terms of the basis $T^{n}$ as $\tau=\sum_{k} c_{k} t_{k}$ and prove that $\operatorname{ad}_{\bullet}(\tau)=\sum_{k} c_{k} \operatorname{ad}_{\bullet}\left(t_{k}\right)=0$ implies that each $c_{k}=0$. We assume that the basis has been sorted in a non-increasing order in terms of the number of subtrees of each element (e.g., $t_{1}$ is the bushy tree). The key observation is that for a tree $t$ with $\ell$ subtrees, one has $\operatorname{ad}_{\bullet}(t)=\left[B_{-}(t), \bullet\right]+\bar{\tau}$ where the first term has $\ell+1$ subtrees and $\bar{\tau} \in \mathcal{T}^{n+1}$ is a sum of trees each having at most $\ell$ subtrees. Furthermore, for two different trees $t$ and $s$, $\left[B_{-}(t), \bullet\right]$ and $\left[B_{-}(s), \bullet\right]$ are different. In particular, the only occurence of the bushy tree of $n+1$ vertices in $\operatorname{ad}_{\bullet}(\tau)$ comes from $c_{1} \operatorname{ad}_{\bullet}\left(t_{1}\right)$ and thus we must have $c_{1}=0$. By induction, assuming that $c_{1}=\cdots=c_{m-1}=0$ we find that the only occurrence of the tree $\left[B_{-}\left(t_{m}\right), \bullet\right]$ in $\operatorname{ad}_{\bullet}\left(\sum_{k \geq m} c_{k} t_{k}\right)$ comes from $c_{m} \operatorname{ad}_{\bullet}\left(t_{m}\right)$ thus $c_{m}=0$. We have proved that all $c_{k}=0$ and therefore ad, is injective on $\mathcal{T}^{n}$.

The Lie bracket on $\mathcal{T}$ can be split into two pieces by introducing the non-associative left pre-Lie product $\triangleright$, see e.g. [9], which is defined on $u, v \in T$ as the sum of all trees obtained by grafting $u$ onto each vertex of $v$. It then holds that $\llbracket u, v \rrbracket=u \triangleright v-v \triangleright u$. For subsequent use, we define $L_{\bullet}, R_{\bullet}$ as the linear maps $L_{\bullet}: u \mapsto \bullet \triangleright u$ and $R_{\bullet}: u \mapsto u \triangleright \bullet=[u]$. Now we can write $\operatorname{ad}_{\bullet}=L_{\bullet}-R_{\bullet}$. The transpose of ad, is denoted ad* $=L_{\bullet}^{*}-R_{\bullet}^{*}$. We have $\operatorname{ad}_{\bullet}^{*}\left(\mathcal{T}^{n+1^{*}}\right)=\mathcal{T}^{n *}$ owing to Lemma 6 . Suppose $t \in T^{n+1}$ and $u \in T^{n}$. We let $r(t, u)$ count the number of leaves that, when removed from $t$, would yield $u$. Clearly we may have $r(t, u)=0$. We have the following formulas for $L_{\bullet}^{*}$ and $R_{\bullet}^{*}$ applied to the dual element $t^{*}$.

$$
L_{\bullet}^{*}\left(t^{*}\right)=\sum_{u \in T^{n}} r(t, u) u^{*} \quad R_{\bullet}^{*}\left(t^{*}\right)= \begin{cases}\bar{t}^{*} & \text { if } t=[\bar{t}] \\ 0 & \text { otherwise }\end{cases}
$$

We may naturally interpret the Butcher product on dual elements as $u^{*} \circ v^{*}:=(u \circ v)^{*}$ for any $u, v \in T$. It is convenient to augment the basis $T$ with the identity element $\emptyset$ of grade 0 , 
such that $\emptyset \cdot \tau=\tau \cdot \emptyset=\tau$ for any $\tau \in \mathcal{T} \oplus \mathbf{R} \emptyset$. We then have $L_{\bullet}^{*}\left(\bullet^{*}\right)=\emptyset^{*}$ and by convention $t \circ \emptyset=-\emptyset \circ t=t$ for any $t \in T .{ }^{3}$

Lemma 7. $\llbracket \mathcal{T}_{H}^{\prime}, \bullet \rrbracket \mathcal{T}_{H}=0$.

Proof. Suppose not. Then there exists $g=F(t), t \in \mathcal{T}_{H}^{\prime}$ satisfying $\llbracket f, g \rrbracket(H)=0$. Then

$$
0=\llbracket f, g \rrbracket(H)=f(g(H))-g(f(H))=f(g(H)) .
$$

That is, $f$ has first integral $g(H)$. But there exist $f$ whose only independent first integral is $H$; in this case, $g(H)=G(H)$ for some scalar function $G$. But $g$ is an elementary differential of $f$ so $G(H)=0$ and $g \in \mathcal{T}_{H}$, a contradiction.

Proof. (A tree-based version) It is enough to prove that for any $n \geq 0$ one has

$$
\forall \tau \in \mathcal{T}^{n}, \quad \operatorname{ad}_{\bullet} \tau \in \mathcal{T}_{H}^{n+1} \Rightarrow \tau \in \mathcal{T}_{H}^{n}
$$

The above condition is equivalent to $\operatorname{Ann}\left(\mathcal{T}_{H}^{n}\right) \subseteq \operatorname{ad}_{\bullet}^{*}\left(\operatorname{Ann}\left(\mathcal{T}_{H}^{n+1}\right)\right)$. Each dual tree appears in at most one basis element (8), this defines a linear surjective $\operatorname{map} \operatorname{ann}_{n}: \mathcal{T}^{n *} \rightarrow \operatorname{Ann}\left(\mathcal{T}_{H}^{n}\right)$, by $\operatorname{ann}_{n}\left(t^{*}\right)=b_{t}$ if $t^{*}$ features in the annihilator basis element $b_{t}$. It suffices to prove that $\operatorname{ann}_{n}\left(t^{*}\right) \in \operatorname{ad}_{\bullet}^{*}\left(\operatorname{Ann}\left(\mathcal{T}_{H}^{n+1}\right)\right)$ for all $t \in T^{n}$. We order $T^{n}$ by decreasing number of subtrees, starting from the bushy tree $t_{1}$ and proceeding by induction. We have $\operatorname{ad}_{\bullet}^{*}\left(\operatorname{ann}_{n+1}\left(t_{1}^{*} \circ \bullet^{*}\right)\right)=$ $\operatorname{ann}_{n}\left(t_{1}^{*}\right)$. For the other trees in $T^{n}$, we use the following relation

$$
\operatorname{ad}_{\bullet}^{*}\left(\operatorname{ann}_{n+1}\left(t^{*} \circ \bullet^{*}\right)\right)=\operatorname{ann}_{n}\left(L_{\bullet}^{*} t^{*} \circ \bullet^{*}+t^{*}\right) .
$$

Suppose that $t^{*}$ has $q$ subtrees. The argument of $\operatorname{ann}_{n}$ on the right hand side equals $m_{t} t^{*}+\tau^{*}$ where $m_{t}$ is a positive integer, and $\tau^{*} \in \mathcal{T}^{n *}$ is a linear combination of dual trees with $q+1$ subtrees. The induction hypothesis asserts that $\operatorname{ann}_{n}\left(\tau^{*}\right) \in \operatorname{ad}_{\bullet}^{*}\left(\operatorname{Ann}\left(\mathcal{T}_{H}^{n}\right)\right)$ and it follows that also $\operatorname{ann}_{n}\left(t^{*}\right) \in \operatorname{Ann}\left(\mathcal{T}_{H}^{n}\right)$.

Lemma 8. $\llbracket \mathcal{T}_{\Omega}^{\prime}, \bullet \rrbracket \cap \mathcal{T}_{\Omega}=0$.

Proof. The vector field $f$ is assumed Hamiltonian, that is, $i_{f} \Omega=d H$ or $d i_{f} \Omega=0$. Suppose the lemma is false. Then there exists $g=F(t), t \in \mathcal{T}_{\Omega}^{\prime}$ such that $\llbracket f, g \rrbracket$ is Hamiltonian, i.e.,

$$
\begin{aligned}
0 & =d i_{\llbracket f, g \rrbracket} \Omega \\
\Rightarrow 0 & =d\left(\mathcal{L}_{f} i_{g} \Omega-i_{g} \mathcal{L}_{f} \Omega\right) \\
\Rightarrow 0 & =d \mathcal{L}_{f} i_{g} \Omega \\
\Rightarrow 0 & =d\left(d i_{f} i_{g} \Omega+i_{f} d i_{g} \Omega\right) \\
\Rightarrow 0 & =d i_{f} \widetilde{\Omega} \text { where } \widetilde{\Omega}:=d i_{g} \Omega=\mathcal{L}_{g} \Omega .
\end{aligned}
$$

That is, the flow of the vector field $f$ preserves both $\Omega$ and $\widetilde{\Omega}$. (In coordinates, $\widetilde{\Omega}=\Omega g^{\prime}+g^{\prime \top} \Omega$.) But there exist $f$ whose flow does not preserve two independent 2 -forms: for example, in $\mathbb{R}^{2}$ with $\Omega=d x \wedge d y, \widetilde{\Omega}=w(x, y) d x \wedge d y$, we would need $\nabla \cdot f=1=w(x, y)$. Regardless of $t$ there will exist $f$ for which $w(x, y) \not \equiv 1$, a contradiction.

\footnotetext{
${ }^{3} \emptyset \circ \emptyset$ remains undefined
} 
Proof. (A tree-based version) As in the proof of Lemma 7 it is enough to show that $\operatorname{Ann}\left(\mathcal{T}_{\Omega}^{n}\right) \subseteq$ ad* $\left(\operatorname{Ann}\left(\mathcal{T}_{\Omega}^{n+1}\right)\right)$. Below, we shall order the basis for $\operatorname{Ann}\left(\mathcal{T}_{\Omega}^{n}\right)$ given in Theorem 3 as $b_{1}, b_{2}, \ldots$ and we use induction. For each $k$ we find $\bar{b}_{k} \in \operatorname{Ann}\left(\mathcal{T}_{\Omega}^{n+1}\right)$ having the property that ad* $\left(\bar{b}_{k}\right)=$ $m_{k} b_{k}+\delta_{k}$ where $m_{k}$ is a positive integer and $\delta_{k}$ is a linear combination of $b_{j}, j<k$, in particular $\delta_{1}=0$. Then $b_{k}=\frac{1}{m_{k}}\left(\operatorname{ad}_{\bullet}^{*}\left(\bar{b}_{k}\right)-\delta_{k}\right) \in \operatorname{ad}_{\bullet}^{*}\left(\operatorname{Ann}\left(\mathcal{T}_{\Omega}^{n+1}\right)\right)$ by the induction hypothesis. Each $b_{k}$ is of the form $u^{*} \circ v^{*}+v^{*} \circ u^{*}$ where $u \in T^{\ell}$ and $v \in T^{n-\ell}$. We order these basis elements non-increasingly in terms of the number of subtrees of $u$, assuming $|u| \geq|v|$. In particular $b_{1}=t_{1}^{*} \circ \bullet^{*}+\bullet^{*} \circ t_{1}$ where $t_{1} \in T^{n-1}$ is the bushy tree. If $b_{k}=u^{*} \circ v^{*}+v^{*} \circ u^{*}$ we let $\bar{b}_{k}=\bar{u}^{*} \circ v^{*}+v^{*} \circ \bar{u}^{*}$ where $\bar{u}=u \circ \bullet$. We have $\operatorname{ad}_{\bullet}^{*}\left(\bar{b}_{1}\right)=(n-1) b_{1}$. We now invoke the formula

$$
\operatorname{ad}_{\bullet}^{*}\left(\bar{u}^{*} \circ v^{*}+v^{*} \circ \bar{u}^{*}\right)=L_{\bullet}^{*} \bar{u}^{*} \circ v^{*}+\bar{u}^{*} \circ L_{\bullet}^{*} v^{*}+L_{\bullet}^{*} v^{*} \circ \bar{u}^{*}+v^{*} \circ L_{\bullet}^{*} \bar{u}^{*} .
$$

But $L_{\bullet}^{*} \bar{u}^{*}=m_{k} u^{*}+\tau^{*}$ where $m_{k}$ is a positive integer, and $\tau$ is a linear combination of trees with precisely one more subtree than $u$. So $L_{\bullet}^{*} \bar{u}^{*} \circ v^{*}+v^{*} \circ L_{\bullet}^{*} \bar{u}^{*}=m_{k} b_{k}+\delta_{k, 1}$ where $\delta_{k, 1}$ is a linear combination of $b_{j}, j<k$. Furthermore, $\bar{u}^{*} \circ L_{\bullet}^{*} v^{*}+L_{\bullet}^{*} v^{*} \circ \bar{u}^{*}=\delta_{k, 2}$ where, since $\bar{u}$ has precisely one more subtree than $u, \delta_{k, 2}$ is a linear combination of $b_{j}, j<k$. Therefore $\operatorname{ad}_{\bullet}^{*}\left(\bar{b}_{k}\right)=m_{k} b_{k}+\delta_{k}$ with $\delta_{k}=\delta_{k, 1}+\delta_{k, 2}$.

Theorem 9. Let $n>2$. As the conjugacy c ranges over $\mathcal{T}_{\Omega}^{\prime}$ with terms of order $<n-1$ fixed, and $\hat{f}$ ranges over $\mathcal{T}_{\Omega}$ with $\hat{f}(\bullet)=1$ and terms of order $<n$ fixed, the order $n$ terms in the conjugate-to-Hamiltonian B-series

$$
\tilde{f}=\hat{f}-\llbracket c, \hat{f} \rrbracket+\frac{1}{2} \llbracket c, \llbracket c, \hat{f} \rrbracket \rrbracket-\ldots
$$

range over the affine space

$$
\mathcal{T}_{\widetilde{\Omega}}^{n}+b
$$

where the linear space

$$
\mathcal{T}_{\widetilde{\Omega}}^{n}:=T_{\Omega}^{n} \oplus \llbracket \mathcal{T}_{\Omega}^{n-1 \prime}, \bullet \rrbracket
$$

and $b \in \mathcal{T}^{n}$ is a constant depending on the lower order terms in $c$ and $\hat{f}$. The space $\mathcal{T}_{\widetilde{\Omega}}^{n}$ is well-defined in the sense that it does not depend on the choice of complement $\mathcal{T}^{n-1 \prime}$. The dimension of $\mathcal{T}_{\widetilde{\Omega}}^{n}$ is

$$
\left|\mathcal{T}_{\widetilde{\Omega}}^{n}\right|=\left|\mathcal{T}_{\Omega}^{n}\right|+\left|\mathcal{T}^{n-1}\right|-\left|\mathcal{T}_{\Omega}^{n-1}\right|
$$

Proof. At order $n$, the only variable terms are $\hat{f}^{n}$ and $\llbracket c^{n-1}, \bullet \rrbracket$; everything else is collected in the constant term $b$. Thus the order $n$ terms in $\tilde{f}$ range over

$$
\mathcal{T}_{\Omega}^{n}+\llbracket \mathcal{T}_{\Omega}^{n-1 \prime}, \bullet+b
$$

By Lemma 8 , this is a direct sum. By Lemma 6 , ad॰ is $1-1$ on $\mathcal{T}^{n-1}$, and hence also $1-1$ on any subspace of $\mathcal{T}^{n-1}$; in particular, on $\mathcal{T}_{\Omega}^{n-1 \prime}$. Thus $\left|\llbracket \mathcal{T}_{\Omega}^{n-1 \prime}, \bullet \rrbracket\right|=\left|\mathcal{T}_{\Omega}^{n-1 \prime}\right|$, giving the dimension result.

Now turn things around and determine the conjugacy $c \in \mathcal{T}_{\Omega}^{\prime}$ from $\tilde{f}$. From Theorem 9 and

$$
\hat{f}=\tilde{f}+\llbracket c, \tilde{f} \rrbracket+\frac{1}{2} \llbracket c, \llbracket c, \tilde{f} \rrbracket \rrbracket+\ldots,
$$


the order $n-1$ terms in $c$ are determined uniquely from the terms of order $n$ terms in $\hat{f}$, of order $\leq n$ in $\tilde{f}$, and of order $<n-1$ in $c$. This determines $c$ uniquely order-by-order.

We now relax the assumption that $c \in \mathcal{T}_{\Omega}^{\prime}$.

Theorem 10. Let $n>4$. Let the conjugacy $c \in \mathcal{T}$ be split into its Hamiltonian and nonHamiltonian parts as $c=c_{\Omega}+c_{\Omega}^{\prime}$. As $c_{\Omega}^{\prime}$ ranges over $\mathcal{T}_{\Omega}^{\prime}$ with terms of order $<n-1$ fixed, $c_{\Omega}$ ranges over $\mathcal{T}_{\Omega}$ with terms of order $<n-3$ fixed, and $\hat{f}$ ranges over $\mathcal{T}_{\Omega}$ with $\hat{f}(\bullet)=1$ and terms of order $<n$ fixed, the order $n$ terms in the conjugate-to-Hamiltonian B-series $\tilde{f}$ range over an affine space with linear part

$$
T_{\Omega}^{n} \oplus\left(\llbracket \mathcal{T}_{\Omega}^{n-1 \prime}, \bullet \rrbracket+c_{\Omega}^{\prime}(\boldsymbol{\ell}) \llbracket T_{\Omega}^{n-3}, \boldsymbol{v} \rrbracket\right)
$$

Proof. Proceeding as in Theorem 9 we get

$$
\begin{aligned}
& \tilde{f}=\hat{f}-\llbracket c_{\Omega}+c_{\Omega}^{\prime}, \hat{f} \rrbracket+\frac{1}{2} \llbracket c_{\Omega}+c_{\Omega}^{\prime}, \llbracket c_{\Omega}+c_{\Omega}^{\prime}, \hat{f} \rrbracket \rrbracket-\ldots \\
&=\left(\hat{f}-\llbracket c_{\Omega}, \hat{f} \rrbracket+\frac{1}{2} \llbracket c_{\Omega}, \llbracket c_{\Omega}, \hat{f} \rrbracket \rrbracket+\ldots\right) \\
&+\left(-\llbracket c_{\Omega}^{\prime}, \hat{f} \rrbracket+\frac{1}{2} \llbracket c_{\Omega}^{\prime}, \llbracket c_{\Omega}^{\prime}, \hat{f} \rrbracket \rrbracket-\ldots\right) \\
& \quad+\frac{1}{2}\left(\llbracket c_{\Omega}, \llbracket c_{\Omega}^{\prime}, \hat{f} \rrbracket \rrbracket+\llbracket c_{\Omega}^{\prime}, \llbracket c_{\Omega}, \hat{f} \rrbracket \rrbracket\right)+\ldots
\end{aligned}
$$

The order $n$ terms in the first parentheses range over $\mathcal{T}_{\Omega}^{n}$ as before. Thus, introducing Hamiltonian terms in $c$ does not not provide any extra degrees of freedom here. The order $n$ terms in the second parentheses range over $\mathcal{T}_{\widetilde{\Omega}}^{n}$ as before, and do not involve $c_{\Omega}$. We thus concentrate on the terms in the third parentheses. The lowest order term in $c_{\Omega}^{\prime}$ is proportional to $\boldsymbol{l}$. $\hat{f}$ has no term proportional to as $\boldsymbol{l}$ is not Hamiltonian. Thus, at order $n, c_{\Omega}$ enters through its order $n-3$ terms as

$$
c_{\Omega}^{\prime}(\boldsymbol{\mathfrak { l }})\left(\llbracket c_{\Omega}^{n-3}, \llbracket \mathfrak{l}, \bullet \rrbracket \rrbracket+\llbracket \mathfrak{\ell}, \llbracket c_{\Omega}^{n-3}, \bullet \rrbracket\right) .
$$

Rearranging using the Jacobi identity, this is

$$
c_{\Omega}^{\prime}(\mathfrak{l})\left(2 \llbracket c_{\Omega}^{n-3}, \boldsymbol{V} \rrbracket-\llbracket \llbracket c_{\Omega}^{n-3}, \mathfrak{q} \rrbracket, \bullet\right) .
$$

The second term lies in $\llbracket T^{n-1}, \bullet \rrbracket$, a space of trees already conjugate to Hamiltonian by $c_{\Omega}^{\prime}$. Only the first term can give anything new. The new space $\llbracket T_{\Omega}^{n-3}, \boldsymbol{V} \rrbracket$ is non-Hamiltonian, by an extension of the proof of Lemma 8. This yields Eq. (13).

Thus, including a Hamiltonian term in the conjugacy potentially increases the dimension of the space of conjugate-to-Hamiltonian B-series from 10 to 11 at order 6 and from 27 to 28 at order 7 (see Table 1). However, to get a definitive result requires the hypothesis that

$$
\llbracket T_{\Omega}^{n-3}, \boldsymbol{V} \rrbracket \cap \llbracket \mathcal{T}_{\Omega}^{n-1 \prime}, \bullet \rrbracket=0
$$

about which we have no information. Regardless, if it happens that $c_{\Omega}^{\prime}(\boldsymbol{\mathfrak { l }})=0$, then we get no new conjugate-to-Hamiltonian terms at all. This will happen if $\tilde{f}(\boldsymbol{o})=0$, so that the secondorder terms do not need to be conjugated, i.e., if the method is second order. In general, if $\tilde{f}$ has order $p$, then including Hamiltonian terms in the conjugacy can only help conjugate terms of order at least $p+2$.

We now consider the conjugate-to-energy-preserving B-series, which are described in precise analogy to Theorem 9; the proof is similar but uses Lemma 7 instead of Lemma 8. 
Theorem 11. Let $n>2$. As the conjugacy c ranges over $\mathcal{T}_{H}^{\prime}$ with terms of order $<n-1$ fixed, and $\hat{f}$ ranges over $\mathcal{T}_{H}$ with $\hat{f}(\bullet)=1$ and terms of order $<n$ fixed, the order $n$ terms in the conjugate-to-energy-preserving B-series

$$
\tilde{f}=\hat{f}-\llbracket c, \hat{f} \rrbracket+\frac{1}{2} \llbracket c, \llbracket c, \hat{f} \rrbracket \rrbracket-\ldots
$$

range over the affine space

$$
\mathcal{T}_{\widetilde{H}}^{n}+b
$$

where the linear space

$$
\mathcal{T}_{\widetilde{H}}^{n}:=T_{H}^{n} \oplus \llbracket \mathcal{T}_{H}^{n-1 \prime}, \bullet \rrbracket
$$

and $b \in \mathcal{T}^{n}$ is a constant depending on the lower order terms in $c$ and $\hat{f}$. The space $\mathcal{T}_{\widetilde{H}}^{n}$ is well-defined in the sense that it does not depend on the choice of complement $\mathcal{T}^{n-1 \prime}$. The dimension of $\mathcal{T}_{\widetilde{H}}^{n}$ is

$$
\left|\mathcal{T}_{\widetilde{H}}^{n}\right|=\left|\mathcal{T}_{H}^{n}\right|+\left|\mathcal{T}^{n-1}\right|-\left|\mathcal{T}_{H}^{n-1}\right|
$$

Theorem 12. For $n>2$,

$$
\mathcal{T}_{\widetilde{H}}^{n}=T_{\Omega}^{n} \oplus T_{H}^{n} .
$$

Proof. The two spaces on the right have zero intersection, and from the dimension results in Theorems 11 and 5(ii) (Eq. (9)), the dimensions on both sides are equal. Also, $T_{H}^{n} \subset T_{\widetilde{H}}^{n}:=T_{H}^{n} \oplus \llbracket T_{H}^{n-1 \prime}, \bullet \rrbracket$. The result was first conjectured using the argument that a Hamiltonian B-series preserves a modified Hamiltonian and hence (see [5], Theorem 5) is conjugate to energy-preserving. Another point of view is to argue that if a B-series has a modified Hamiltonian, then the condition to be energy-preserving is that the coefficient of each elementary Hamiltonian vanishes, and these conditions are in 1-1 correspondence with the Hamiltonian trees. However, we present here instead a constructive proof that resolves an element of $\mathcal{T}_{\widetilde{H}}^{n}$ into a unique sum of Hamiltonian and energy-preserving terms.

Recall that to each rooted tree $\left[t_{1}, \ldots, t_{n}\right]$ is associated the elementary Hamiltonian $H^{(n)}\left(F\left(t_{1}\right), \ldots, F\left(t_{n}\right)\right)$. We let $X_{G}$ denote the Hamiltonian vector field with Hamiltonian $G$, and $X_{t}$ the combination of trees such that $F\left(X_{t}\right)$ is the Hamiltonian vector field whose elementary Hamiltonian is associated with $t$, see [11, Lemma 9.7].

We claim that for all $t \in \mathcal{T}, \llbracket t, \bullet-X_{[t]} \in \mathcal{T}_{H}$. That is, the Hamiltonian component of $\llbracket t, \bullet \in \mathcal{T}_{\widetilde{H}}$ is $X_{[t]}$, and the energy-preserving component is the remainder. We establish the equivalent statement for elementary differentials. Let $g=F(t)$. The elementary differential associated with $\llbracket t, \bullet \rrbracket$ is $\llbracket g, f \rrbracket$, and the elementary differential associated with $X_{[t]}$ is $X_{H^{\prime}(g)}=$ $X_{g(H)}$. Using $f=X_{H}$ and $f(H)=0$ gives

$$
\begin{aligned}
\left(\llbracket g, f \rrbracket-X_{g(H)}\right)(H) & =g(f(H))-f(g(H))-X_{g(H)}(H) \\
& =-X_{H}(g(H))-X_{g(H)}(H) \\
& =-\{H, g(H)\}-\{g(H), H\} \\
& =0 .
\end{aligned}
$$

The claim can also be established in terms of trees. We have $\llbracket t, \bullet \rrbracket=[t]-L_{\bullet}(t)$ where $L_{\bullet}(t)$ is considered as a sum of $|t|$ terms some of which might be identical. Similarly, we note that $X_{[t]}$ 
equals $[t]$ plus an alternating sum of $|t|$ terms corresponding to the root being moved to each of the nodes other than the root of $[t]$. In this way, we may ignore the symmetry coefficients. First, note that the term $[t]$ appears in both $\llbracket t, \bullet \rrbracket$ and $X_{[t]}$ and thus cancels. Then, select a node $x$ of $t$ and consider the three trees $t, t^{\bullet}$ and $t^{x}$ in the picture below. $t^{\bullet}$ is the term in $L_{\bullet}(t)$ obtained by adding a leaf to $x$, whereas $t^{x}$ is obtained from $[t]$ by shifting the root $s$ places to $x$. This term appears with the sign $(-1)^{s}$ in $X_{[t]}$. Adding the terms together results in $t^{\bullet}+(-1)^{s} t^{x}$, but this is precisely an element of the set $S$ of Theorem 4 and is therefore energy-preserving. We conclude that $\llbracket t, \bullet \rrbracket-X_{[t]}$ is a sum of $|t|$ elements from the set $S$ and thus energy-preserving.

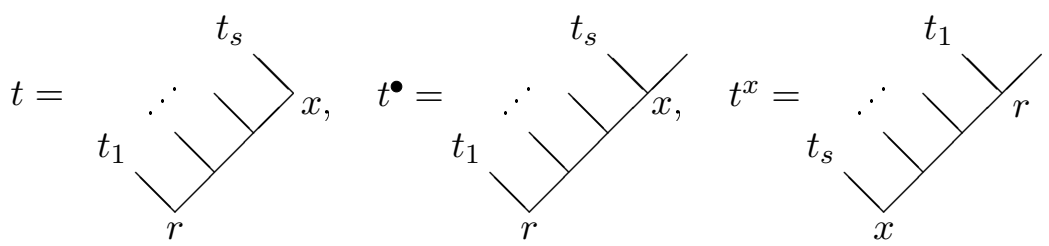

It is curious to note that $\llbracket g, f \rrbracket-X_{g(H)}$ is energy-preserving for any vector field $g$ when $f$ is Hamiltonian with respect to any symplectic structure, not just $g$ an elementary differential of $f$ and $\Omega$ constant, as we have been assuming everywhere else. This decomposition of vector fields in $\operatorname{ad} X_{H}$ into energy-preserving and Hamiltonian parts is explored further in [13].

Example 3. Consider $t=\mathcal{V}$. We have

$$
\llbracket \mathscr{V}, \rrbracket=\Psi-\boldsymbol{\Psi}-3
$$

The Hamiltonian vector field associated with $[t]$ is

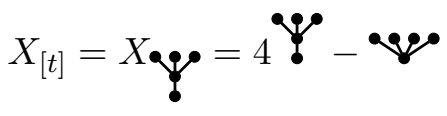

and thus

$$
\llbracket \mathscr{v}, \rrbracket=X \mathfrak{\varphi}-3(\boldsymbol{Y}+\boldsymbol{\psi})
$$

where the term in parentheses is energy-preserving (being one of the basis elements of Theorem 5).

The next space to consider is $\mathcal{T}_{\widetilde{\Omega}}$.

Theorem 13. $\mathcal{T}_{\widetilde{\Omega}} \subset \mathcal{T}_{\widetilde{H}}$.

Proof. From Theorem 12, it suffices to decompose any $t \in \mathcal{T}_{\widetilde{\Omega}}$ into a sum of Hamiltonian and energy-preserving B-series. The same proof as in Theorem 12 achieves this, for $\mathcal{T}_{\widetilde{\Omega}}=$ $\mathcal{T}_{\Omega} \oplus \llbracket \mathcal{T}_{\Omega}^{\prime}, \bullet \rrbracket$. Only the second term needs to be considered, but the proof that $\llbracket t, \bullet \rrbracket-X_{[t]} \in \mathcal{T}_{H}$ works for any $t$. We do not get equality (as in Theorem 12) because the dimensions do not match.

We now ask what new subspaces can be created from the 4 constructed so far. 
Theorem 14. (i) From the four naturally-defined subspaces of B-series, namely $\mathcal{T}_{\Omega}^{n}, \mathcal{T}_{H}^{n}$, $\mathcal{T}_{\widetilde{\Omega}}^{n}$, and $\mathcal{T}_{\widetilde{H}}^{n}$, precisely one new subspace can be constructed using the natural subspace operations of intersection and sum. This is $\mathcal{T}_{\widetilde{\Omega}}^{n} \cap \mathcal{T}_{H}^{n}$, the energy-preserving conjugateto-Hamiltonian B-series.

(ii) $\mathcal{T}_{\widetilde{\Omega}}^{n} \cap \mathcal{T}_{H}^{n}$ is isomorphic to $\mathcal{T}_{\Omega}^{n-1 \prime}$, and an isomorphism is given by the map

$$
\mathcal{T}_{\Omega}^{n-1 \prime} \rightarrow \mathcal{T}_{\widetilde{\Omega}}^{n} \cap \mathcal{T}_{H}^{n}, \quad t \mapsto \llbracket t, \bullet \rrbracket-X_{[t]}
$$

(iii) Its dimension is

$$
\left|\mathcal{T}_{\widetilde{\Omega}}^{n} \cap \mathcal{T}_{H}^{n}\right|=\left|\mathcal{T}^{n-1}\right|-\left|\mathcal{T}_{\Omega}^{n-1}\right|=\operatorname{dim} \operatorname{Ann} \mathcal{T}_{\Omega}^{n-1}
$$

(iv) There are B-series that are energy-preserving and conjugate-to-Hamiltonian, but are not the (reparameterized) flow of the original differential equation.

Proof.

(i) From 4 subspaces and 2 (symmetric) operations, 12 subspaces may be formed. From Theorems 12 and 13, all but one of these are either empty or are one of the 4 listed subspaces; the other one is $\mathcal{T}_{\widetilde{\Omega}}^{n} \cap \mathcal{T}_{H}^{n}$.

(ii) Recall

$$
\mathcal{T}_{\widetilde{\Omega}}^{n}=\mathcal{T}_{\Omega}^{n} \oplus \llbracket \mathcal{T}_{\Omega}^{n-1 \prime}, \bullet \rrbracket \subset \mathcal{T}_{\widetilde{H}}^{n}=\mathcal{T}_{\Omega}^{n} \oplus \mathcal{T}_{H}^{n} .
$$

Every element of $\mathcal{T}_{\widetilde{\Omega}}^{n}$ is the sum of a Hamiltonian and an energy-preserving B-series, and we can calculate $\mathcal{T}_{\widetilde{\Omega}}^{n} \cap \mathcal{T}_{H}^{n}$ by projecting $\mathcal{T}_{\widetilde{\Omega}}^{n}$ onto $\mathcal{T}_{H}^{n}$. Let $s+\llbracket t, \bullet \in \mathcal{T}_{\widetilde{\Omega}}^{n}$, i.e., $s \in \mathcal{T}_{\Omega}^{n}$, $t \in \mathcal{T}_{\Omega}^{n-1 \prime}$. The projections of $\mathcal{T}_{\widetilde{\Omega}}^{n}$ onto $\mathcal{T}_{\Omega}^{n}$ and $\mathcal{T}_{H}^{n}$ are given by

$$
\operatorname{pr}_{\Omega}(s+\llbracket t, \bullet \rrbracket)=s+X_{[t]}, \quad \operatorname{pr}_{H}(s+\llbracket t, \bullet \rrbracket)=\llbracket t, \bullet \rrbracket-X_{[t]}
$$

because the right hand sides have the correct sum and lie in the correct, triviallyintersecting spaces. This yields the result.

(iii) Follows directly from (ii).

(iv) The flow of any element of $\mathcal{T}_{\widetilde{\Omega}} \cap \mathcal{T}_{H}$ has the required properties, and the dimension of $\mathcal{T}_{\widetilde{\Omega}}^{n} \cap \mathcal{T}_{H}^{n}$ is positive for $n>2$.

Note that the isomorphism in Eq. (15) is not canonically defined in terms of the natural basis of trees because it depends on the choice of complement of $\mathcal{T}_{\Omega}^{n}$.

Note that Theorem 14 does not provide a constructive approach to determining whether B-series methods that are both energy-preserving as well as conjugate-to-symplectic exist. However, the large dimensions of $\mathcal{T}_{\widetilde{\Omega}}^{n} \cap \mathcal{T}_{H}^{n}$ (see Table 1) for large $n$ are tantalizing!

We collect the previous results in the following theorem.

Theorem 15. The (Hasse) order diagram under inclusion [8] for the linear spaces $\mathcal{T}^{n}, \mathcal{T}_{H}^{n}$, $\mathcal{T}_{\Omega}^{n}, \mathcal{T}_{\widetilde{H}}^{n}$, and $\mathcal{T}_{\widetilde{\Omega}}^{n} \cap \mathcal{T}_{H}^{n}$ for $n>2$ is 


\begin{tabular}{|r|rrrrrrrrrr|}
\hline order & 1 & 2 & 3 & 4 & 5 & 6 & 7 & 8 & 9 & 10 \\
\hline$\left|\mathcal{T}^{n}\right|$ & 1 & 1 & 2 & 4 & 9 & 20 & 48 & 115 & 286 & 719 \\
$\left|\mathcal{T}_{\Omega}^{n}\right|$ & 1 & 0 & 1 & 1 & 3 & 4 & 11 & 19 & 47 & 97 \\
$\left|\mathcal{T}_{H}^{n}\right|$ & 1 & 0 & 1 & 1 & 5 & 9 & 29 & 68 & 189 & 484 \\
$\left|\mathcal{T}_{\widetilde{\Omega}}^{n}\right|$ & 1 & 0 & 2 & 2 & 6 & 10 & 27 & 56 & 143 & 336 \\
$\left|\mathcal{T}_{\widetilde{H}}^{n}\right|$ & 1 & 0 & 2 & 2 & 8 & 13 & 40 & 87 & 236 & 581 \\
$\left|\mathcal{T}_{\widetilde{\Omega}}^{n} \cap T_{H}^{n}\right|$ & 1 & 0 & 1 & 1 & 3 & 6 & 16 & 37 & 96 & 239 \\
\hline
\end{tabular}

Table 1: Dimensions of the linear spaces spanned by the rooted trees and their 5 natural subspaces.

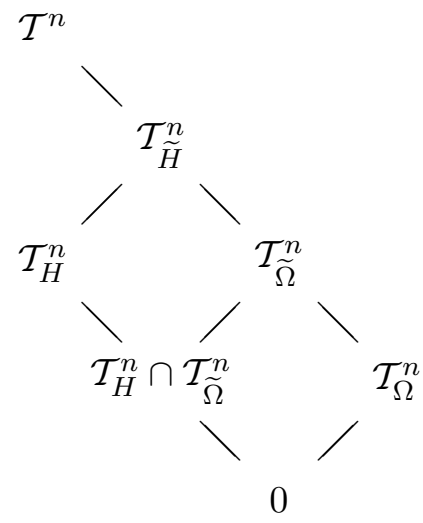

and their dimensions up to order 10 are as given in Table 1 . For $n=1$ all these spaces are equal to $\operatorname{span}(\bullet)$, while for $n=2$ we have $\mathcal{T}^{2}=\operatorname{span}(\boldsymbol{\bullet})$ and $\mathcal{T}_{H}^{2}=\mathcal{T}_{\Omega}^{2}=\mathcal{T}_{\widetilde{H}}^{2}=\mathcal{T}_{\widetilde{\Omega}}^{2}=\mathcal{T}_{\widetilde{\Omega}}^{2} \cap \mathcal{T}_{\widetilde{H}}^{2}=0$.

Example 4. The subspaces of the 9-dimensional space $\mathcal{T}^{5}$ can be represented schematically as follows:

$$
\begin{array}{ccccccccc}
1 & 2 & 3 & 4 & 5 & 6 & 7 & 8 & 9 \\
\widetilde{H} & \widetilde{H} & \widetilde{H} & \widetilde{H} & \widetilde{H} & \widetilde{H} & \widetilde{H} & \widetilde{H} & ? \\
\Omega & \Omega & \Omega & H & H & H & H & H & ? \\
\widetilde{\Omega} & \widetilde{\Omega} & \widetilde{\Omega} & \widetilde{\Omega} & \widetilde{\Omega} & \widetilde{\Omega} & ? & ? & ?
\end{array}
$$

where '?' indicates that we know of no natural subspace for the complementary spaces. The order-5 terms of the B-series of the modified vector field of the AVF method (2) lie in $\mathcal{T}_{H}^{5}$ (because it is energy-preserving) but not in $\mathcal{T}_{\widetilde{\Omega}}^{5}$ (see [4]).

The number of rooted trees $\left|\mathcal{T}^{n}\right|$ is asymptotic to $\beta n^{-3 / 2} \alpha^{-n}$ as $n \rightarrow \infty$, where $\alpha \approx 0.3383$ and $\beta \approx 0.4399$ are Otter's tree enumeration constants [18], and the number of free trees is asymptotic to $\left|F T^{n}\right| \sim \beta^{\prime} n^{-5 / 2} \alpha^{-n}$ where $\beta^{\prime}=2 \pi \beta^{3} \approx 0.5349$. Defining $\gamma=2 \pi \beta^{2}$ and substituting these into the dimension results in Theorems 3, 5, 9 and 11 yields the asymptotic growth rates in Table 2.

Acknowledgements. RIM acknowledges the support of the Marsden Fund of the Royal Society of New Zealand. GRWQ is the recipient of an Australian Research Council Professorial Fellowship. BO and EC acknowledge the support of the Norwegian Research Council. We are 


\begin{tabular}{|l|ll|}
\hline$V$ & $|V| /\left|\mathcal{T}^{n}\right|, n \rightarrow \infty$ & $\approx$ \\
\hline $\mathcal{T}_{\Omega}^{n}$ & $\frac{\gamma}{n}$ & $\frac{1.2160}{n}$ \\
$\mathcal{T}_{H}^{n}$ & $1-\frac{\gamma}{\alpha n}$ & $1-\frac{3.5942}{n}$ \\
$\mathcal{T}_{\widetilde{\Omega}}^{n}$ & $\alpha+\frac{3 \alpha+2 \gamma(1-\alpha)}{2 n}$ & $0.3383+\frac{1.3121}{n}$ \\
$\mathcal{T}_{\widetilde{H}}^{n}$ & $1-\frac{(1-\alpha) \gamma}{\alpha n}$ & $1-\frac{2.3782}{n}$ \\
$\mathcal{T}_{\widetilde{\Omega}}^{n} \cap \mathcal{T}_{H}^{n}$ & $\alpha+\frac{\alpha(3-2 \gamma)}{2 n}$ & $0.3383+\frac{0.0961}{n}$ \\
\hline
\end{tabular}

Table 2: Asymptotic growth of the dimensions of 5 subspaces of B-series, relative to the dimension of the space of all B-series of order $n$, as $n \rightarrow \infty$, correct up to factors $1+\mathcal{O}(1 / n) .$. Here $\gamma=2 \pi \beta^{2}$, and $\alpha$ and $\beta$ are Otter's tree enumeration constants.

grateful to Kurusch Ebrahimi-Fard, David McLaren, Hans Munthe-Kaas, and Will Wright for useful discussions. In particular, McLaren performed the computations needed in Example 2, and Ebrahimi-Fard pointed us to the underlying pre-Lie algebra product in the operators $L$. and $R_{\bullet}$.

\section{References}

[1] A Iserles, G R W Quispel, and P S P Tse, B-series methods cannot be volume-preserving. BIT Numerical Mathematics, 47(2): 351Đ378, 2007.

[2] J C Butcher, An algebraic theory of integration methods, Math. Comput. 26 (1972), 79-106.

[3] M P Calvo and J M Sanz-Serna, Canonical B-series, Numer. Math. 67, (1994), 161-175.

[4] E Celledoni, R I McLachlan, D I McLaren, B Owren, G R W Quispel, and W M Wright, Energy-preserving Runge-Kutta methods, M2AN, to appear.

[5] P Chartier, E Faou, and A Murua, An algebraic approach to invariant preserving integrators: The case of quadratic and Hamiltonian invariants, Numer. Math. 103 (2006), $575-590$.

[6] P Chartier and A Murua, Preserving first integrals and volume forms of additively split systems, IMA Journal of Numerical Analysis 27 (2007), 2007, 381-405.

[7] G J Cooper, Stability of Runge-Kutta methods for trajectory problems, IMA J. Numer. Anal. 7 (1987) 1-13.

[8] B A Davey and H A Priestley, Introduction to Lattices and Order, Cambridge University Press, Cambridge, 2002. 
[9] K Ebrahimi-Fard and D Manchon, A Magnus- and Fer-Type Formula in Dendriform Algebras, Found. Comput. Math. 9 (2009) 295-316.

[10] E Faou, E Hairer, and T-L Pham, Energy conservation with non-symplectic methods: examples and counter-examples, BIT 44 (2004) 699-709.

[11] E Hairer, C Lubich, and G Wanner, Geometric Numerical Integration: StructurePreserving Algorithms for Ordinary Differential Equations, Springer, Berlin, 2nd ed., 2006.

[12] E Hairer, R I McLachlan, and R D Skeel, On energy conservation of the simplified Takahashi-Imada method, M2AN, to appear.

[13] R I McLachlan, The structure of a set of vector fields on Poisson manifolds, J. Phys. A 42 (2009) 142001 (3 pp).

[14] R I McLachlan, G R W Quispel, and N Robidoux, Geometric integration using discrete gradients, Phil. Trans. Roy. Soc. A 357 (1999), 1021-1046.

[15] R I McLachlan, G R W Quispel, and P S P Tse, Linearization-preserving self-adjoint and symplectic integrators, BIT 49(1) (2009), 177-197.

[16] R I McLachlan, G R W Quispel, and G S Turner, Numerical integrators that preserve symmetries and reversing symmetries, SIAM J. Numer. Anal. 35 (1998) 586-599.

[17] A Murua, Formal series and numerical integrators, Part I: Systems of ODEs and symplectic integrators, Appl. Numer. Math. 29 (1999) 221-251.

[18] R Otter, The number of trees, Ann. Math. 49(3) (1948), 583-599.

[19] G R W Quispel and D I McLaren, A new class of energy-preserving numerical integration methods, J. Phys. A 41 (2008) 045206 (7pp).

[20] J E Scully, A search for improved numerical integration methods using rooted trees and splitting, MSc Thesis, La Trobe University, 2002.

[21] L F Shampine, Conservation laws and the numerical solution of ODEs, Comput. Math. Appl. 12B (1986) 1287-1296.

\section{Appendix. Conjugate B-series as vector bundles}

We recapitulate the construction of Section 3 by presenting a geometric description of the manifold of conjugate B-series as a vector bundle.

Suppose $U$ and $V$ are graded subspaces of $\mathcal{T}$ so that

$$
U=\bigoplus_{n>0} U^{n}, \quad V=\bigoplus_{n>0} V^{n}
$$


with $U^{n}=\mathcal{T}^{n} \cap U$ and $V^{n}=\mathcal{T}^{n} \cap V$. The elements of $U$ and $V$ are $B$-series. We want to study $B$-series belonging to $\mathcal{T}$, which are conjugations of elements in $V$ by elements in $U$, or more precisely, we want to characterize the set

$$
\mathcal{M}=\left\{w=\exp \left(-\operatorname{ad}_{u}\right) v, u \in U, v \in V\right\}
$$

where

$$
w=\exp \left(-\operatorname{ad}_{u}\right) v=v-\llbracket u, v \rrbracket+\frac{1}{2} \llbracket u, \llbracket u, v \rrbracket \rrbracket+\cdots
$$

Since $w$ is a nonlinear function of $u$, we cannot expect $\mathcal{M}$ to be a linear subspace of $\mathcal{T}$. Neither is it clear how $\mathcal{M}$ can be written as a graded space. For instance, even if $u$ and $v$ intersect only with a finite number of homogeneous components $\left(U^{n}, V^{n}\right), w$ will typically have an infinite number of components.

We consider the quotient spaces

$$
G^{n}=\mathcal{T} / \bigoplus_{k>n} \mathcal{T}^{k}
$$

so that in $G^{n}$ we distinguish only between $B$-series which differ in their first $n$ components. We can also naturally identify elements of $G^{n}$ with elements of $\mathcal{T}^{1} \oplus \cdots \oplus \mathcal{T}^{n}$. We let $\mathcal{P}^{n}: \mathcal{T} \rightarrow G^{n}$ be the canonical projection mapping an element of $\mathcal{T}$ to the equivalence class it belongs to. It now makes sense to study the manifolds $\mathcal{M}^{n}=\mathcal{P}_{n} \mathcal{M} \subseteq G^{n}$, and in particular to look at their dimensions. We introduce the space $\mathcal{B}^{n} \subseteq G^{n}$

$$
\mathcal{B}^{n}=\left\{w=\mathcal{P}_{n} \exp \left(-\operatorname{ad}_{u}\right) v, u \in \bigoplus_{k \leq n-2} U^{k}, v \in \bigoplus_{k \leq n-1} V^{k}\right\}
$$

or in words, we consider only those series we can get by using terms up to order $n-2$ in $u$ and $n-1$ in $v$. It is useful to observe that the previously defined $\mathcal{M}^{n}$ can be interpreted in a similar way, namely as

$$
\mathcal{M}^{n}=\left\{w=\mathcal{P}_{n} \exp \left(-\operatorname{ad}_{u}\right) v, u \in \bigoplus_{k \leq n-1} U^{k}, v \in \bigoplus_{k \leq n} V^{k}\right\},
$$

this is because the terms of order greater than $n-1$ in $u$ and greater than $n$ in $v$ do not contribute to the terms of order less than or equal to $n$ in $w$. The reason is that the commutator respects the grading of $\mathcal{T}$, in the sense that $\tau \in \mathcal{T}^{k}, \tau^{\prime} \in \mathcal{T}^{\ell} \Rightarrow \llbracket \tau, \tau^{\prime} \rrbracket \in \mathcal{T}^{k+\ell}$. We can consider elements of $\mathcal{M}^{n}$ expressed as the finite sum

$$
w=\sum_{k \leq n} v^{k}-\sum_{k+\ell \leq n} \llbracket u^{\ell}, v^{k} \rrbracket+\frac{1}{2} \sum_{k+\ell+m \leq n} \llbracket u^{\ell}, \llbracket u^{m}, v^{k} \rrbracket \rrbracket+\cdots
$$

where $u^{k} \in U^{k}, v^{k} \in V^{k}$ being the order $k$ components of $u$ and $v$ respectively.

To proceed, we shall assume that $V$ contains the element •, and consider only series in $V$ such that $v^{1}=$ •. We now consider for each $n$ the triple $\left(\mathcal{M}^{n}, \mathcal{B}^{n}, \pi\right)$ where $\pi: \mathcal{M}^{n} \rightarrow \mathcal{B}^{n}$ is 
the projection obtained by simply removing the $n$-1-component of $u$ and the $n$-component of $v$. Precisely, if

$$
w=\mathcal{P}_{n} \exp \left(-\operatorname{ad}_{u}\right) v, \quad u=\sum_{k=1}^{n-1} u^{k}, \quad v=\bullet+\sum_{k=2}^{n} v^{k}
$$

then

$$
\pi w=\mathcal{P}_{n} \exp \left(-\operatorname{ad}_{\bar{u}}\right) \bar{v}, \quad \bar{u}=\sum_{k=1}^{n-2} u^{k}, \quad \bar{v}=\bullet+\sum_{k=2}^{n-1} v^{k} .
$$

The triple $\left(\mathcal{N}^{n}, \mathcal{B}^{n}, \pi\right)$ forms a vector bundle with total space $\mathcal{M}^{n}$, base space $\mathcal{B}^{n}$ and projection $\pi$. The typical fiber is $F^{n}=\pi^{-1}(x)$, and by construction this space is obtained simply by considering all terms of (16) which depend only on the $n$-1-component of $u$ and the $n$ component of $v$,

$$
F^{n}=V^{n}+\llbracket U^{n-1}, \bullet \rrbracket .
$$

Using the identification of $G^{n}$ with $\mathcal{T}^{1} \oplus \cdots \oplus T^{n}$ it is easy to see that $\operatorname{dim} \mathcal{B}^{n}=\operatorname{dim} \mathcal{M}^{n-1}$, thus,

$$
\operatorname{dim} \mathcal{M}^{n}=\operatorname{dim} \mathcal{B}^{n}+\operatorname{dim} F^{n}=\operatorname{dim} \mathcal{M}^{n-1}+\operatorname{dim} F^{n}
$$

so that the dimension of $\mathcal{M}^{n}$ is obtained by summing up the dimensions of each $F^{k}$ for $k=1, \ldots, n$. 